\title{
Neonatal screening for four lysosomal storage diseases with a digital microfluidics platform: Initial results in Brazil
}

\author{
Eurico Camargo Neto ${ }^{1}$, Jaqueline Schulte ${ }^{1}$, Jamile Pereira ${ }^{1}$, Heydy Bravo ${ }^{2,3}$, Claudio Sampaio-Filho ${ }^{4}$ and \\ Roberto Giugliani ${ }^{2,3,5}$ \\ ${ }^{1}$ Centro de Triagem, Porto Alegre, RS, Brazil. \\ ${ }^{2}$ Serviço de Genética Médica, Hospital de Clínicas de Porto Alegre, Porto Alegre, RS, Brazil. \\ ${ }^{3}$ Programa de Pós-graduação em Genética e Biologia Molecular, Universidade Federal do Rio Grande do \\ Sul, Porto Alegre, RS, Brazil. \\ ${ }^{4}$ Intercientífica, São José dos Campos, SP, Brazil. \\ ${ }^{5}$ Departamento de Genética, Universidade Federal do Rio Grande do Sul, Porto Alegre, RS, Brazil.
}

\begin{abstract}
We describe the initial results of a neonatal screening program for four lysosomal storage diseases (MPS I, Pompe, Gaucher and Fabry) using the digital microfluidics methodology. The method successfully identified patients previously diagnosed with these diseases and was used to test dried blood spot samples obtained from 10,527 newborns aged 2 to 14 days. The digital microfluidic technology shows potential for a simple, rapid and high-throughput screening for these four diseases in a standard neonatal screening laboratory.
\end{abstract}

Keywords: Lysossomal storage diseases, neonatal screening, digital microfluidics, Brazil.

Received: July 26, 2017; Accepted: November 17, 2017.

Neonatal screening for lysosomal storage diseases (LSDs) has been gaining considerable interest as a result of the new policies to promote early diagnosis of rare diseases, the development of new screening methods, and the availability of enzyme replacement and other specific therapies for several LSDs. Also, LSDs are not considered too rare anymore, with a combined incidence for the around 50 LSDs estimated to be around 1/7,000 (Meikle et al., 1999; Poorthuis et al., 1999).

LSDs have a wide spectrum of clinical signs and symptoms, with overlapping findings across different diseases and with most disorders presenting large variability (Gieselmann, 2005). This makes the diagnosis particularly difficult, especially as it needs to be confirmed with sophisticated biochemical and genetic tools (Wang et al., 2011). For these reasons there is often a delay in the diagnosis of LSDs (Vieira et al., 2008). This delay may influence the outcome of treatment, already available for several of these conditions (Giugliani et al., 2016).

The development of high-throughput protocols with multiplexing capabilities for use with dried blood spot (DBS) samples has facilitated the establishment of NBS

Send correspondence to Roberto Giugliani. Serviço de Genética Médica, Hospital de Clinicas de Porto Alegre, Rua Ramiro Barcelos 2350, 90035-903 Porto Alegre, RS, Brazil. E-mail: rgiugliani@ hcpa.edu.br. programs for LSDs around the world (Spada et al., 2006; Hwu et al., 2009; Hopkins et al., 2015).

We present here the initial results obtained with a fluorimetric digital microfluidics (DMF) platform used for the neonatal screening of four LSDs [Mucopolysaccharidosis I (MPS I), Gaucher, Fabry, and Pompe diseases].

A DMF platform was used to assay the activities of $\alpha$-L- iduronidase (IDUA), to screen for MPS I; acid $\alpha$-glucosidase (GAA), to screen for Pompe; acid $\beta$-glucosidase (GBA), to screen for Gaucher; and acid $\alpha$-galactosidase (GLA), to screen for Fabry in the newborn's samples.

We used one workstation with four digital microfluidic instruments to run a multiplexed fluorometric enzymatic assay platform as described previously (Sista et al., 2011; Sista et al., 2013). All necessary hardware and reagents for GAA, GBA, GLA, and IDUA were supplied by Baebies Inc (Durham, NC, USA).

DBS samples from patients previously diagnosed with MPS I, Gaucher, Pompe or Fabry diseases were initially tested to check the capability of the method in identifying the respective enzyme deficiencies (Table 1).

DBS samples were then collected from 10,527 newborns aged 2 to 14 days of life, randomly selected for testing among the cards routinely received by the Neonatal Screening Center, based in Porto Alegre, Brazil. Samples from newborns were spotted on the filter paper and shipped 
at room temperature to the reference laboratory, arriving there within 24 to 72 hours . Tests were performed no more than 48 hours after sample arrival.

The DMF assay protocol to determine the enzyme activity in a multiplex format took less than four hours, allowing three runs per day (eventually, one overnight) and yielded 152 results in each run (total, 456 per day) when using one working station with four fluorimeters in parallel. Overall coefficient of variation (CV) values between cartridges, days, instruments, and operators ranged from 4 to $22 \%$. Linearity correlation coefficients were $\geq 0.98$ for all assays.

Cutoff values of $5.1 \mu \mathrm{mol} / \mathrm{L} / \mathrm{h}$ for IDUA, $5.9 \mu \mathrm{mol} / \mathrm{L} / \mathrm{h}$ for GAA, $3.9 \mu \mathrm{mol} / \mathrm{L} / \mathrm{h}$ for $\mathrm{GBA}$, and $5.7 \mu \mathrm{mol} / \mathrm{L} / \mathrm{h}$ for GLA were established during a preliminary phase using 1,000 samples from the cards routinely collected and DBS specimens from patients with confirmed LSDs previously diagnosed. These cut-off values were not very different from the ones established by Hopkins et al. (2015), using the same method $(4.0,8.0,4.5$, and $5.5 \mu \mathrm{mol} / \mathrm{L} / \mathrm{h}$, respectively).

The method correctly discriminated nine samples of affected patients ( 3 from MPS I, 2 from Pompe, 2 from Gaucher and 2 from Fabry cases) previously diagnosed, from samples of normal subjects. Further information on cutoffs and results in known patients is displayed in Table 1.

Four samples out of the 10,527 tested showed activity below the cutoff and were further investigated until a final conclusion was established. These cases were studied by another group and eventually classified as pseudodeficiency (low activity of enzyme without generation of storage and with no clinical consequences, usually found in patients who present mutations already known as related to pseudodeficiency) of $\alpha$-L-iduronidase carriership for MPS I ( 1 case), pseudodeficiency of $\alpha$-glucosidase ( 1 case) and carriership for Gaucher disease (1 case) (Bravo et al., 2017).

The availability of effective therapies for several LSDs (Aronovich et al., 2015) and the evidence that early introduction of therapy may bring a better outcome (Gabrielli et al., 2016) make neonatal screening for these conditions an option to be considered. This is especially true as several high-throughput platforms became available to perform tests in a large number of samples at a low cost per assay (Matern et al., 2015).

In the present report we demonstrated that DMF was efficient in identifying samples from patients previously diagnosed with MPS I, Gaucher, Fabry and Pompe diseases. Although we cannot discard that false negatives could occur, this possibility is considered very small, as affected patients usually have extremely low enzyme activities, and all affected patients tested were identified by the method in the validation process (data not shown). The usefulness of this
Table 1 - Averages for each enzyme activity in normal subjects, cut-off values and results in confirmed cases previously diagnosed.

\begin{tabular}{|c|c|}
\hline MPS-1 & Results $(\mu \mathrm{mol} / \mathrm{L} / \mathrm{h})$ \\
\hline \multicolumn{2}{|l|}{ Samples $(n=9864)$} \\
\hline IDUA average & 17.1 \\
\hline calculated IDUA cut-off ( $30 \%$ ) & 5.1 \\
\hline \multicolumn{2}{|l|}{ Positive samples $(\mathrm{n}=3$ ) } \\
\hline Patient 1 & 3.4 \\
\hline Patient 2 & 4.9 \\
\hline Patient 3 & 3.7 \\
\hline FABRY & Results $(\mu \mathrm{mol} / \mathrm{L} / \mathrm{h})$ \\
\hline \multicolumn{2}{|l|}{ Samples (n=9988) } \\
\hline GLA average & 18.9 \\
\hline calculated GLA cut-off $(30 \%)$ & 5.7 \\
\hline \multicolumn{2}{|l|}{ Positive samples ( $\mathrm{n}=2$ ) } \\
\hline Patient 1 & 4.2 \\
\hline Patient 2 & 4.7 \\
\hline Patient 3 & 3.6 \\
\hline POMPE & Results $(\mu \mathrm{mol} / \mathrm{L} / \mathrm{h})$ \\
\hline \multicolumn{2}{|l|}{ Samples $(n=9560)$} \\
\hline GAA average & 19.8 \\
\hline calculated GAA cut-off $(30 \%)$ & 5.9 \\
\hline \multicolumn{2}{|l|}{ Positive samples ( $\mathrm{n}=2$ ) } \\
\hline Patient 1 & 3.4 \\
\hline Patient 2 & 3.1 \\
\hline GAUCHER & Results $(\mu \mathrm{mol} / \mathrm{L} / \mathrm{h})$ \\
\hline \multicolumn{2}{|l|}{ Samples $(\mathrm{n}=9878)$} \\
\hline GBA average & 13 \\
\hline calculated GBA cut-off ( $30 \%)$ & 3.9 \\
\hline \multicolumn{2}{|l|}{ Positive samples (n-2) } \\
\hline Patient 1 & 3.3 \\
\hline Patient 2 & 3.5 \\
\hline
\end{tabular}

platform was already reported by Hopkins et al. (2015), who detected 1/1,618 newborns affected by one of these four diseases in 43,686 samples evaluated. We identified four samples among the 10,527 DBS from newborns tested with a low enzyme activity $(1 / 2,631)$. Although subsequent testing informed that these were not true LSDs, all these cases had true low enzyme activity, which was detected by the screening program using the DMF method.

This is the first report of neonatal screening for multiple LSDs conducted in Brazil. It is important to mention that this program was performed in a standard clinical biochemistry laboratory, indicating that, in addition to be efficient and robust, the platform is suitable to less sophisticated laboratories, as are most of the laboratories in Latin America. The successful use of this strategy to simultaneously measure four enzyme activities indicates that this 
method could be an option for Brazil and other developing countries.

\section{Acknowledgments}

The authors are grateful to Babies, Inc for providing the hardware and reagents to run this program.

\section{References}

Aronovich EL and Hackett PB (2015) Lysosomal storage disease: Gene therapy on both sides of the blood-brain barrier. Mol Genet Metab 114:83-93.

Bravo H, Camargo-Neto E, Schulte J, Pereira J, Sampaio-Fiho C, Bittencourt F, Sebastião F, Bender F, Magalhães APS, Guidobono $\mathrm{R}$ et al. (2017) Investigation of newborns with abnormal results in a newborn screening program for four lysosomal storage diseases in Brazil. Mol Genet Metab Reports 12:92-97.

Gabrielli O, Clarke LA, Ficcadenti, A, Santoro L, Zampini L, Volpi, N and Coppa GV (2016) 12 year follow up of enzyme-replacement therapy in two siblings with attenuated mucopolysaccharidosis I: The important role of early treatment. J Med Genet 17:1-7.

Gieselmann V (2005) What can cell biology tell us about heterogeneity in lysosomal storage diseases? Acta Paediatr 94:8086.

Giugliani R, Federen A, Vairo F, Vanzella C, Pasqualim G, Silva LMR, Giugliani L, Boder APK, Souza CFM, Matte U and Baldo G (2016) Emerging drugs for the treatment of mucopolysaccharidoses. Exp Opinion Emerg Drugs 21:9-26.

Hopkins PV, Campbell C, Klug T, Rogers S, Raburn-Miller J and Kiesling J (2015) Lysosomal storage disorder screening implementation: findings from the first six months of full population pilot testing in Missouri. J Pediatr 166:172-177.

Hwu WL, Chien YH, Lee NC, Chiang SC, Dobrovolny R, Huang AC, Yeh HY, Chao MC, Lin SJ, Kitagawa T et al. (2009) Newborn screening for Fabry disease in Taiwan reveals a high incidence of the later-onset GLA mutation c.936+919G $>$ A (IVS4+919G > A). Hum Mutat 30:1397-1405.

Matern D, Gavrilov D, Oglesbee D, Raymond K, Rinaldo P and Tortorelli S (2015) Newborn screening for lysosomal storage disorders. Semin Perinatol 39:206-16.

Meikle PJ, Hopwood JJ, Clague AE and Carey WF (1999) Prevalence of lysosomal storage disorders. JAMA 281:249-254.

Poorthuis, BJHM, Wevers RA, Kleijer WJ, Groener JEM, De Jong JGN, Van Weely S, Niezen-Koning KE and Van Diggelen OP (1999) The frequency of lysosomal storage diseases in The Netherlands. Hum Genet 105:151-156.

Sista RS, Eckhardt AE, Wang T, Graham C, Rouse JL, Norton SM, Srinivasan V, Pollack MG, Tolun AA, Bali D et al. (2011) Digital microfluidic platform for multiplexing enzyme assays: Implications for lysosomal storage disease screening in newborns. Clin Chem 57:1444-1451.

Sista RS, Wang T, Wu N, Graham C, Eckhardt A, Winger T, Srinivasan V, Bali D, Millington DS and Pamula VK (2013) Multiplex newborn screening for Pompe, Fabry, Hunter, Gaucher, and Hurler diseases using a digital microfluidic platform. Clin Chim Acta: 424:12-18.

Spada M, Pagliardini S, Yasuda M, Tukel T, Thiagarajan G, Sakuraba H, Ponzone A and Desnick RJ (2006) High incidence of later-onset Fabry disease revealed by newborn screening. Am J Hum Genet 79:31-40.

Vieira T, Schwartz IVD, Muñoz V, Pinto L, Steiner C, Ribeiro M, Boy R, Ferraz V, De Paula A, Kim C et al. (2008) Mucopolysaccharidoses in Brazil: What happens from birth to biochemical diagnosis? Am J Med Genet 146A:1741-1747.

Wang RY, Bodamer OA, Watson MS and Wilcox WR (2011) Lysosomal storage diseases: Diagnostic confirmation and management of presymptomatic individuals. Genet Med $13: 457-484$

Associate Editor: Carlos F.M. Menck

License information: This is an open-access article distributed under the terms of the Creative Commons Attribution License (type CC-BY), which permits unrestricted use, distribution and reproduction in any medium, provided the original article is properly cited. 